\title{
High mechanical Q-factor measurements on calcium fluoride at cryogenic temperatures
}

\author{
R. Nawrodt ${ }^{\mathrm{a}}$, A. Zimmer, T. Koettig, S. Nietzsche, M. Thürk, W. Vodel, and P. Seidel \\ Friedrich-Schiller-Universität Jena, Institut für Festkörperphysik, Helmholtzweg 5, 07743 Jena, Germany
}

Received: 1 September 2006 / Accepted: 12 January 2007

Published online: 22 February 2007 - (c) EDP Sciences

\begin{abstract}
Mechanical $Q$-factor measurements of single crystalline calcium fluoride $\mathrm{CaF}_{2}$ bulk material are presented. Resonant modes between 27 and $100 \mathrm{kHz}$ were investigated on a cylindrical test sample $(\varnothing 75 \mathrm{~mm} \times 75 \mathrm{~mm})$. For selected modes low temperature measurements of the mechanical $Q$-factor were done within a temperature range from 5 to $300 \mathrm{~K}$. For the $Q$-measurements a ring-down technique was used. The substrate was suspended as a pendulum by means of a tungsten wire loop. The highest $Q$-factor has been achieved around $60 \mathrm{~K}$ with $3 \times 10^{8}$ which is the highest $Q$-factor reported on $\mathrm{CaF}_{2}$ up to now.
\end{abstract}

PACS. 43.40.-r Structural acoustics and vibration $-43.60 .+\mathrm{d}$ Acoustic signal processing $-62.30 .+\mathrm{d}$ Mechanical and elastic waves; vibrations - 04.80.Nn Gravitational wave detectors and experiments

\section{Introduction}

Interferometric gravitational wave detectors like GEO600 [1], LIGO [2], VIRGO [3], or TAMA [4] are among instruments with the highest sensitivity for lengths changes ever developed. The design resolution of the displacement of the British-German detector GEO600 is better than $h \approx 10^{-22}$. Ground based gravitational wave detectors have a detection bandwidth from several $10 \mathrm{~Hz}$ up to a few $\mathrm{kHz}$. Different kinds of noise are limiting the sensitivity of current detectors. Thermal noise is one of the fundamental noise sources of the optical components. Several approaches exist to overcome this limitation. One of the most promising attempts is cooling the crucial parts of the optics to minimize thermal noise [5]. In addition it is possible to influence the spectral distribution of the thermal noise. By the use of high- $Q$ materials most of the thermal noise occurs around the mechanical resonances of the test mass. These resonances are in the range of several $10 \mathrm{kHz}$ and so well above the detection band of an interferometric gravitational wave detector. Accordingly, the thermal noise level within the detection band is lowered. Therefore, low loss materials are needed providing a high mechanical $Q$-factor at low temperatures. Candidates for substrate materials are high-purity single-crystals as silicon [6] or sapphire [7]. Especially sapphire is planned to be used as a test mass material in the Japanese Large Scale Cryogenic Gravitational Wave Telescope (LCGT, [8]).

\footnotetext{
a e-mail: ronny.nawrodt@uni-jena.de
}

During the recent years calcium fluoride has become an interesting optical material [9] due to its high transmittance from $0.13 \mu \mathrm{m}$ to $12 \mu \mathrm{m}$ [10] and low absorption $(1 \mathrm{ppm} / \mathrm{cm}$, measured by the Perugia group within the STREGA project [11]). In addition, calcium fluoride shows a high laser damage threshold $[12,13]$ which allows the use of higher laser powers in the interferometers. This is required to lower the photon shot noise of the system.

The $Q$-factor of a system at a given frequency is related to the losses $\phi$ at the same frequency by [14]:

$$
\phi=\frac{1}{Q}
$$

The mechanical loss $\phi$ is directly linked to the thermal noise of the system by the fluctuation-dissipation theorem [15]. The thermal noise power spectrum $\overline{x^{2}}$ is given by $[16]$ :

$$
\overline{x^{2}} \propto \frac{T}{Q}=T \times \phi
$$

for a one-dimensional harmonic oscillator far away from its resonance. $T$ is the sample temperature and $\phi$ the mechanical loss.

The mechanical $Q$-factor is a strongly temperature dependent parameter [17]. Therefore, it is necessary to investigate this dependence in order to optimize the detectors. During the recent years, only a few measurements concerning the internal mechanical losses of calcium fluoride were published. Yan et al. [18] measured the $Q$-factor of calcium fluoride bulk material at room temperature. They found a highest value of $4.5 \times 10^{7}$. Yamamoto et al. [19] 


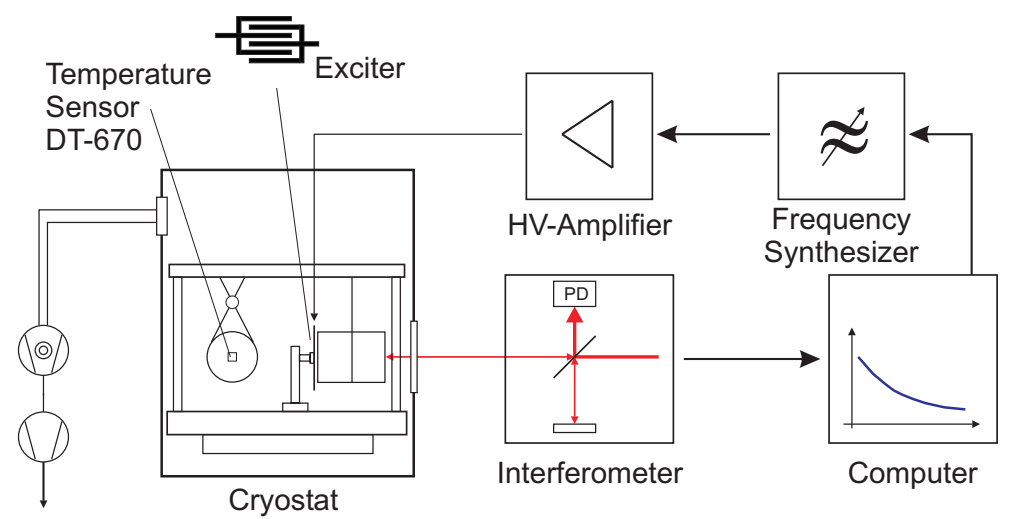

Fig. 1. Schematic overview of the measuring setup. The test substrate is suspended as a pendulum in a special built cryostat [20]. A second test sample (here 90 degrees rotated for clarity) is symmetrically placed in the probe volume for thermometry using a Lakeshore DT-670 temperature sensor. The test sample is excited by means of a comb-like electrostatic actuator which is fed by a high-voltage amplifier. The sample vibration is read-out by a Michelson-like interferometer. The whole setup is computer controlled.

investigated the $Q$-factor of a thin calcium fluoride disk $(\varnothing 100 \mathrm{~mm} \times 3 \mathrm{~mm})$ at discrete low temperatures around $10 \mathrm{~K}$ and $70 \mathrm{~K}$. They found a top value of $9 \times 10^{7}$ at $12 \mathrm{~K}$.

In this paper we present detailed $Q$-factor measurements between 5 and $300 \mathrm{~K}$ on calcium fluoride bulk material. Different suspension geometries were used in order to distinguish between losses arising from the test sample and losses from the suspension.

\section{Experimental setup}

\subsection{Overview}

The experiments were carried out in a special built cryostat to determine the mechanical $Q$-factor at different temperatures. The cryostat consists of a probe chamber $(\varnothing 300 \mathrm{~mm} \times 500 \mathrm{~mm}$ ) which is temperature-stabilized between 5 and $325 \mathrm{~K}$. The temperature stability is better than $0.25 \mathrm{~K} / \mathrm{h}$. Avoiding gas-damping, the probe volume can be evacuated to a pressure lower than $10^{-3} \mathrm{~Pa}$ using a turbo-molecular pump. Details of the cryostat are given in [20]. Figure 1 shows a schematic overview of the measuring setup.

The test sample was suspended as a pendulum by means of a tungsten wire loop [21]. It is necessary to use a thin wire to lower the influence of the suspension material as much as possible. A $75 \mu \mathrm{m}$ wire was chosen although a $50 \mu \mathrm{m}$ wire would have been sufficient to withstand the mirror weight. The thinner wire cuts into the substrate material due to the low hardness of $\mathrm{CaF}_{2}$. This reduced the $Q$-factor by a factor of approximately 5 . Before suspending the substrate the wire was polished with a $3 \mu \mathrm{m}$ diamond paste [22].

A second test sample was placed in the probe chamber for thermometry. A temperature sensor DT-670 from LakeShore [23] was attached to that substrate. The setup is symmetric for both substrates and therefore the temperature of both substrates should be the same. This has been confirmed in a separate test run.
The substrate is excited to resonance vibrations by means of an electrostatic driving structure. The structure is fed by a high (up to $1600 \mathrm{~V}$ ) AC voltage with the resonant frequency [24]. The substrate vibration is recorded by a Michelson-like interferometer [25]. The distance between the beamsplitter and the substrate is compared to a reference arm. The difference of the lengths can be directly read out of the interferometer with a resolution of approximately $0.1 \mathrm{~nm}$ in a frequency range from 0.1 to $500 \mathrm{kHz}$. Using an FFT algorithm the vibrational amplitude at the resonances is obtained from the interferometer signal. After reaching some nanometers of amplitude the exciter is switched off and the subsequent ring-down is recorded by a computer. The amplitude follows an exponential law:

$$
A=A_{0} \cdot e^{-t / \tau}
$$

with the initial amplitude $A_{0}$, the time $t$ and the characteristic ring-down time $\tau$. The mechanical $Q$-factor of the system is connected to this ring-down time by [14]:

$$
Q=\pi f_{0} \tau
$$

with the resonant frequency $f_{0}$.

The measurement error of a ring-down experiment is better than $2 \%$ for the fit algorithm and lower than $1 \%$ for the measurement of the resonant frequency. All measurements were carried out several times. However, the repeatability of these measurements limits an over-all error of the $Q$-values to about $10 \% \ldots 20 \%$.

The temperature was stabilized for each measurement and the probe chamber evacuated to a pressure lower than $10^{-3} \mathrm{~Pa}$. Afterwards, the high voltage was switched on and the modes of interest were measured. For each temperature step this took between 15 and 90 minutes depending on the ring-down time of the modes. Typically, the probe temperature was stable to $0.3 \mathrm{~K}$ within this time frame. 
Table 1. Sample parameters of the $\mathrm{CaF}_{2}$-single crystals. All thermal and elastic properties are listed for $300 \mathrm{~K}$. The references given for the different parameters contain the full temperature dependence down to $4.2 \mathrm{~K}$.

\begin{tabular}{ll}
\hline Parameter & Value \\
\hline manufacturer & Schott $[26]$ \\
orientation & $(100)$ \\
polishing & Hellma-Optik $[27]$ \\
flatness & $\lambda / 4(\lambda=633 \mathrm{~nm})$ \\
diameter & $75 \mathrm{~mm}$ \\
thickness & $75 \mathrm{~mm}$ \\
density $\rho$ & $3.18 \mathrm{~g} / \mathrm{cm}^{3}[26]$ \\
Poisson's ratio $\sigma$ & $0.28[28]$ \\
thermal expansion coefficient $\alpha$ & $1.8 \times 10^{-5} \mathrm{~K}^{-1}[29]$ \\
thermal conductivity $\kappa$ & $9.71 \frac{\mathrm{W}}{\mathrm{m} \cdot \mathrm{K}}[30]$ \\
specific heat capacity C & $890 \frac{\mathrm{kg} \cdot \mathrm{K}}{28,31]}$ \\
elastic constants & $1.64 \times 10^{11} \mathrm{~N} / \mathrm{m}^{2}[28]$ \\
$\quad C_{11}$ & $5.30 \times 10^{10} \mathrm{~N} / \mathrm{m}^{2}[28]$ \\
$C_{12}$ & $3.37 \times 10^{10} \mathrm{~N} / \mathrm{m}^{2}[28]$ \\
\hline$C_{44}$ &
\end{tabular}

\subsection{Test samples}

The measurements were made on $\mathrm{CaF}_{2}$-single crystals manufactured by Schott [26]. The sample parameters are given in Table 1. The crystal's [100] direction is parallel to the cylindrical axis. All substrate surfaces (front, back, circumference, and bevels) were polished to an optical quality by Hellma-Optik Jena [27].

The substrate for the $Q$-measurement and the thermometry substrate were identical in shape and polishing.

\section{Results}

\subsection{Measurements at $320 \mathrm{~K}$}

In a first run the substrate was suspended by means of a polished $75 \mu \mathrm{m}$ tungsten wire. Several spectral scans between $20 \mathrm{kHz}$ and $100 \mathrm{kHz}$ were performed to screen for possible modes for the low temperature investigations. Due to the interferometer technique it is not possible to measure all modes of the substrate. Only modes with a sufficient amplitude at the read-out point of the interferometer can be detected. 47 modes could be observed with a maximum amplitude between $0.2 \mathrm{~nm}$ and $24 \mathrm{~nm}$ using the maximum excitation voltage. Table 2 gives the measured values of selected modes. The mode number is the consecutive number of the mode observed in our experiment. The measured resonant frequencies were compared to a finite-element-analysis using ANSYS [32] to identify the modes and their shape. These calculations were carried out using the room temperature values of the elastic constants. The measured data were recorded during the initial vacuum pumping. During this phase a warm-up to $320 \mathrm{~K}$ takes place to support the cleaning of the probe chamber surface. The calculated resonant frequencies agree within $2 \%$ with the measured values.
Table 2. Compilation of selected modes. Numerical calculations were done by the use of ANSYS [32] with the set of material parameters at $300 \mathrm{~K}$ (see Tab. 1) and an accuracy of $\pm 0.5 \mathrm{~mm}$ in diameter and length. The calculated resonant frequencies agree within $2 \%$ with the measured ones although the measurements were taken between 300 and $320 \mathrm{~K}$ during a warm-up period.

\begin{tabular}{clll}
\hline $\begin{array}{c}\text { Consecutive } \\
\text { mode number }\end{array}$ & \multicolumn{2}{l}{ Resonant frequency $[\mathrm{Hz}]$} & measured \\
measured & calculated & -factor \\
\hline 1 & 26957.6 & 26931 & $1.3 \times 10^{6}$ \\
2 & 29791.4 & 29684 & $5.6 \times 10^{6}$ \\
4 & 31796.7 & 32464 & $5.0 \times 10^{6}$ \\
8 & 40310.2 & 40856 & $5.6 \times 10^{6}$ \\
9 & 41301.7 & 40950 & $1.1 \times 10^{7}$ \\
16 & 50476.3 & 50595 & $1.3 \times 10^{7}$ \\
18 & 51862.3 & 51594 & $7.0 \times 10^{6}$ \\
19 & 52484.6 & 52622 & $7.4 \times 10^{6}$ \\
20 & 57421.1 & 57562 & $5.1 \times 10^{6}$ \\
24 & 63889.8 & 63888 & $3.6 \times 10^{6}$ \\
26 & 67310.4 & 66468 & $2.8 \times 10^{5}$ \\
28 & 70097.7 & 70944 & $2.2 \times 10^{6}$ \\
\hline
\end{tabular}

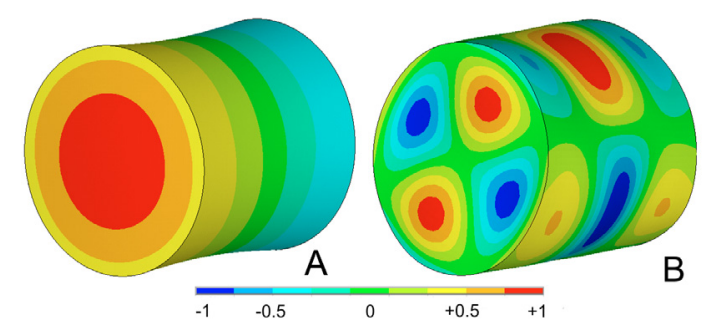

Fig. 2. Calculated mode shapes of two modes using ANSYS [32]. A: fundamental drum mode (number 9 in Tab. 2), B: higher mode (number 26 in Tab. 2). The color indicates the displacement along the cylindrical axis of the given point. The colored chart represents the displacement normalized to the highest value of the particular mode.

It is obvious that the $Q$-factor changes more than one order of magnitude for the selected modes. In fact, modes with $Q$-factors of less than $10^{6}$ were also observed among the modes given in Table 2 . By analyzing the corresponding mode shapes known from the numerical calculations it is apparent that only modes with a small displacement at the suspension points have high measured $Q$-factors. Figure 2 gives two examples of calculated mode shapes. Mode A - the so called fundamental drum mode - has low displacement around the midplane. The $Q$-factor of this mode was $1.1 \times 10^{7}$. In contrast, mode $\mathrm{B}$ has large fluctuations around the midplane. The $Q$-factor of this mode is lower and was found to be $2.8 \times 10^{5}$.

The highest $Q$-factor was observed for the fundamental drum mode (mode 9 in Tab. 2). The value of $1.1 \times 10^{7}$ is 4 times lower than the maximum $Q$-value measured by Yan et al. [18] who used a different suspension geometry and a $50 \mu \mathrm{m}$ tungsten wire for the suspension loop. In our experiment a $75 \mu \mathrm{m}$ tungsten wire was used as explained above. The lower $Q$-factor is supposed to be due to the 


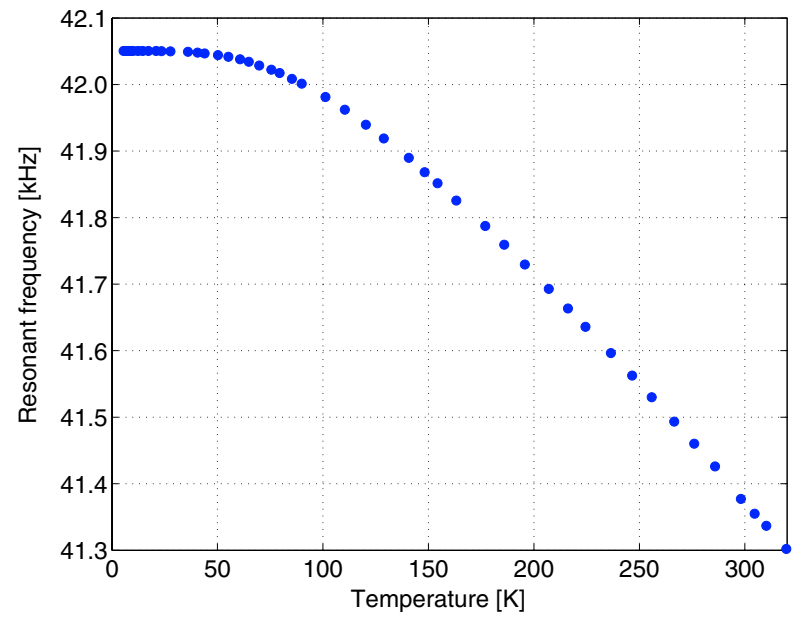

Fig. 3. Measured temperature dependence of the resonant frequency of the fundamental drum mode (mode number 9, see Fig. 2A for modeshape). All temperatures are within an error of about $0.3 \mathrm{~K}$, the resonant frequencies to better than $0.1 \%$.

different suspension or different purity of the substrate material.

\subsection{Cryogenic Q-measurements}

Due to the long measuring times only three modes were selected for the low temperature experiment. The gravitational wave detector thermal noise will be dominated by noise contributions of modes with large displacement in the center of the front surface. Therefore, the fundamental drum mode plays an important role. The temperature dependence of the fundamental drum mode frequency is shown in Figure 3. The resonant frequency increased monotonically while cooling the sample to $5 \mathrm{~K}$. The frequency shift was $750 \mathrm{~Hz}$ in total. Thus, temperature changes of the substrate can be monitored without attaching a sensor to the substrate using this dependency.

Figure 4 gives the $Q$-factor of the fundamental drum mode (mode number 9) among two other selected modes (mode number 8 and 20 from Tab. 2) during the cool down cycle. A measurement cycle as explained in Section 2.1 was carried out each $10 \mathrm{~K}$ between $300 \mathrm{~K}$ and $100 \mathrm{~K}$. Below $100 \mathrm{~K}$ the temperature steps were smaller.

The maximum $Q$-factor has been observed between 60-70 K. The fundamental drum mode provides the highest $Q$-factor reaching $3.2 \times 10^{8}$ at $64 \mathrm{~K}$. Below this temperature the $Q$-factor decreases reaching a level of $5.8 \times 10^{7}$ at $5.5 \mathrm{~K}$.

Yamamoto et al. presented in [19] a $Q$-factor of several $10^{7}$ below $12 \mathrm{~K}$ which corresponds pretty well to our observations although the test samples have completely different geometries (Yamamoto et al.: $\varnothing 100 \mathrm{~mm} \times 3 \mathrm{~mm}$, here: $\varnothing 75 \mathrm{~mm} \times 75 \mathrm{~mm})$. Also a decreasing $Q$-factor with decreasing temperature below $15 \mathrm{~K}$ could be observed. At temperatures around $77 \mathrm{~K}$ the $Q$-value reported by Yamamoto of $\approx 10^{6}$ was limited by thermoelastic damping due to the geometry of the test sample. Our measure-

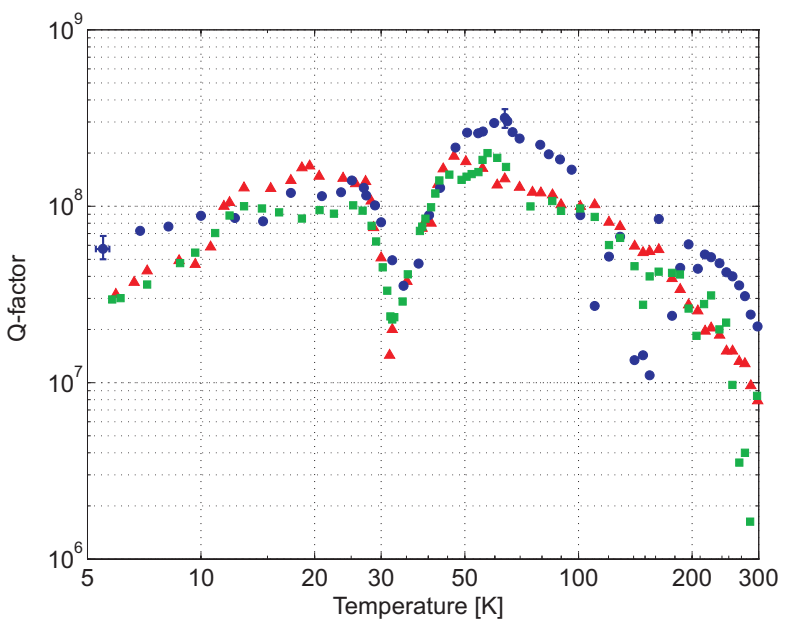

Fig. 4. Measured values of the mechanical $Q$-factor of the $\mathrm{CaF}_{2}$ test sample for three different modes. Blue circles: $41302 \mathrm{~Hz}$ (mode number 9), red triangles: $40310 \mathrm{~Hz}$ (mode number 8), green squares: $57421 \mathrm{~Hz}$ (mode number 20). All resonant frequencies at $300 \mathrm{~K}$. Orientation: (100), dimension: $\varnothing 75 \mathrm{~mm} \times 75 \mathrm{~mm}$, suspension: tungsten wire loop $(\varnothing 75 \mu \mathrm{m})$. All temperatures are measured with an accuracy of better than $0.3 \mathrm{~K}$. The $Q$-factor has an error of about $10 \ldots .20 \%$ due to reproducibility. For the lowest temperature and the highest $Q$-factor error bars are plotted exemplarily for the $41302 \mathrm{~Hz}$ mode.

ments led to $Q$-values up to a factor of 10 higher at this temperature.

Between $100 \mathrm{~K}$ and $200 \mathrm{~K}$ dips could be observed. These dips can be explained by resonances with the suspension wire loop. The resonant frequency is strongly dependent on temperature (see Fig. 3). If the substrate frequency coincides with a higher harmonic of the suspension wire energy is efficiently transferred from the substrate to the wire. This would cause additional damping resulting in a dip in the $Q$-dependence on temperature.

Two modes were measured in a second test run using other suspension parameters to clarify the suspension's influence. Figure 5 shows the measured $Q$-values within a temperature range from $60 \mathrm{~K}$ to $300 \mathrm{~K}$. A shift of the resonant dips to other temperatures could be observed compared to previous measurements. The resonances now occur at $144 \mathrm{~K}$ for mode number 8 and at $198 \mathrm{~K}$ for the mode number 9 . This corresponds to a resonant frequency of $41010.6 \mathrm{~Hz}$ for the first and $41720.5 \mathrm{~Hz}$ for the second mode obtained from the measured frequency-temperature dependency. The difference fits very well to the separation of the suspension wire resonances which was calculated to be $680 \mathrm{~Hz}$. Additionally, mode number 9 has two more dips at around $135 \mathrm{~K}$ and $250 \mathrm{~K}$. The seperation of the different resonant dips of mode 9 is approximately $200 \mathrm{~Hz}$. One can conclude that the resonances are based on an interaction between substrate and suspension. Furthermore, it can be predicted from these results that the measurements below $100 \mathrm{~K}$ are not limited by suspension resonances. The resonant frequency shift from $100 \mathrm{~K}$ to $5 \mathrm{~K}$ is not sufficiently large for the next higher order violin mode of the suspension to appear (for the $680 \mathrm{~Hz}$ and 


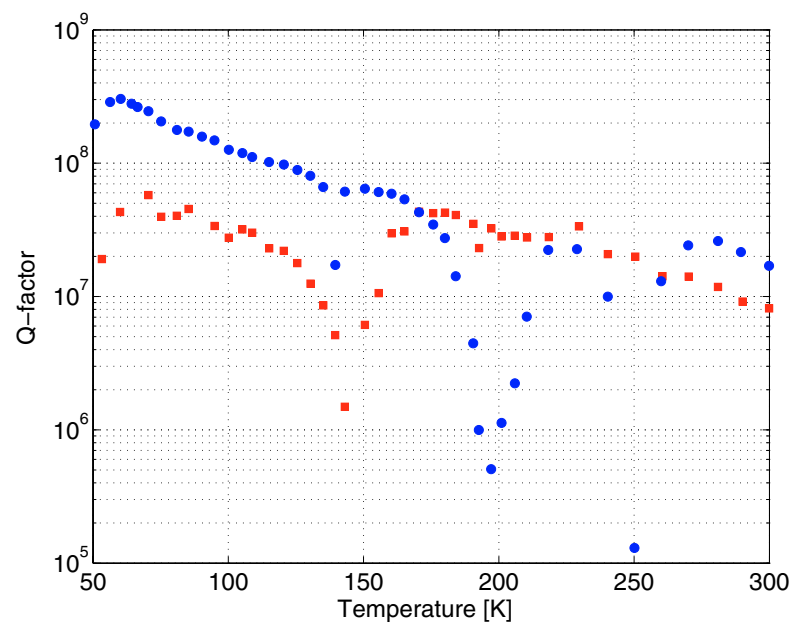

Fig. 5. Measured $Q$-factors of two modes with a different suspension from that of Figure 4. The temperatures of the resonant dips changed compared to the first suspension setup. The resonant frequencies belonging to these temperatures are $41010.6 \mathrm{~Hz}$ for mode 8 (red squares) and $41720.5 \mathrm{~Hz}$ for mode 9 (blue circles) respectively. The difference of these frequencies corresponds well to the separation of the suspension violin modes. In addition mode 9 has some more resonant dips (at about $135 \mathrm{~K}$ and about $250 \mathrm{~K}$ ).

the $200 \mathrm{~Hz}$ separation). This indicates that the measurements at low temperatures were not limited by suspension resonances for the investigated modes.

A strong resonance effect can be observed between 30 and $40 \mathrm{~K}$ for all modes in Figure 4 . Here the mechanical $Q$-factor drops to approximately $2 \times 10^{7}$. The resonant frequencies at the $Q$-factor minima are $41230 \mathrm{~Hz}$ (mode 8), $42050 \mathrm{~Hz}$ (mode 9), and $58750 \mathrm{~Hz}$ (mode 20). This effect seems to be an intrinsic effect of the substrate. External influences from the measuring setup on the test sample could be excluded by former measurements on silicon test samples which were identically in shape. These measurements led to no additional damping between 30 and $40 \mathrm{~K}$. Similar resonant effects were observed in crystalline quartz samples [33]. Here, impurities introduced relaxation effects in the material. Further measurements are needed to reveal the origin of this resonance effects in calcium fluoride. For example, relaxation effects would exhibit a special shift of the resonant dip with frequency. The current results do not provide exact values of the position of the lowest $Q$-value due to the large temperature steps. $Q$-factor measurements of more modes covering a wide frequency range with small temperature steps are needed to investigate these effects more in detail.

The thermal noise of a mirror like substrate was calculated (see Fig. 6). The internal thermal noise is dependent on the mechanical properties (and therefore the mechanical $Q$-factor), the beam radius and beam profile of the laser used in the detector. The calculation was done using Levin's direct approach [34] assuming a frequency independent loss (also known as structural damping). This is only a coarse approximation. Several experiments revealed that the $Q$-factor is usually a frequency dependent value.

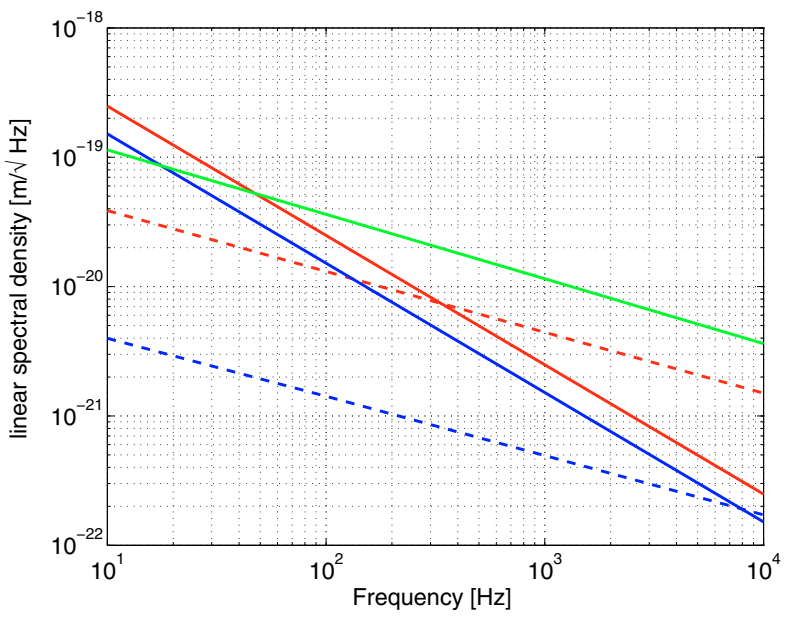

Fig. 6. Displacement linear spectral density of a fused silica and a calcium fluoride substrate. The calculation was done using Levin's direct approach [34]. Beam diameter: $45 \mathrm{~mm}$. Green solid: fused silica $\left(Q=3.8 \times 10^{6}, 300 \mathrm{~K}\right.$, currently used in GEO600 [36], total thermal noise), red: $\mathrm{CaF}_{2}\left(Q=2 \times 10^{7}\right.$, $300 \mathrm{~K}$, dashed: structural contribution, solid: thermoelastic contribution), blue: $\mathrm{CaF}_{2}\left(Q=3 \times 10^{8}, 64 \mathrm{~K}\right.$, dashed: structural contribution, solid: thermoelastic contribution). Thermoelastic noise dominates for the calcium fluoride substrates within the detection band.

Penn et al. proposed a model for the frequency dependence of fused silica [35]. In lack of a suitable model for calcium fluoride a frequency independent $Q$-factor was assumed. The results for the linear spectral density of calcium fluoride are compared to the values of a fused silica mirror at $300 \mathrm{~K}$ currently used in GEO600. The $Q$-factors of calcium fluoride at $300 \mathrm{~K}$ and $64 \mathrm{~K}$ were obtained from our measurements. The $Q$-factor for fused silica at room temperature was assumed to be $Q=3.8 \times 10^{6}[36]$ as measured at the real test masses of GEO600. However, much higher $Q$-factors were demonstrated at a higher quality fused silica (see e.g. [37] with $2 \times 10^{7}$ ). The calculation reveals a possible noise improvement of one order of magnitude between the fused silica at room temperature currently used and calcium fluoride at $64 \mathrm{~K}$ for the internal thermal noise.

Nevertheless another kind of thermal noise needs to be taken into account arising from the thermal properties of the test mass material. This kind of noise is called thermoelastic noise and is dependent on the beam radius and the thermal properties of the material. Braginski et al. calculated the thermoelastic power spectral density for an infinite half space [38] and a gaussian laser beam:

$$
S_{x}^{2}(f)=\frac{\sqrt{2}}{\pi^{5 / 2}} \cdot \frac{k_{B} T^{2} \alpha^{2}(1+\sigma)^{2} \kappa}{\rho^{2} C^{2} r_{0}^{3} f^{2}},
$$

with the substrate temperature $T$, the thermal expansion coefficient $\alpha$, the Poisson's ratio $\sigma$, the thermal conductivity $\kappa$, the mass density $\rho$, the specific heat capacity $C$, the beam radius $r_{0}$ (1/e of the maximum intensity), and the frequency $f$. Liu and Thorne investigated the influence of a finite size of the test mass [39]. They found a 
correction factor in the order of unity which was neglected for the calculations presented in this paper. From Figure 6 it is obvious that thermoelastic noise dominates within the detection band at $300 \mathrm{~K}$ as well as at $64 \mathrm{~K}$ for calcium fluoride with the beam parameters currently used in GEO600. Using a larger beam diameter could overcome this problem because the thermoelastic noise decreases faster with the beam diameter than the contribution from the internal thermal noise. Another possibility would be the use of a non-Gaussian beam shape. Although, using calcium fluoride instead of fused silica would improve the detector noise at frequencies above approximately $50 \mathrm{~Hz}$ especially at low temperatures. Fused silica - as being an amorphous material - can not be used at low temperatures due to its low mechanical $Q$-factor (huge Debye-peak around $40 \mathrm{~K}[5])$.

\section{Conclusion}

Systematic low temperature mechanical $Q$-factor measurements of a calcium fluoride single crystal were done within a temperature range from $5 \mathrm{~K}$ up to $300 \mathrm{~K}$. The highest $Q$-factor has been observed for the fundamental drum mode around $65 \mathrm{~K}$ as $3 \times 10^{8}$. The interaction between the test sample and the suspension was carefully investigated. It was demonstrated that below $100 \mathrm{~K}$ no resonant effects between the substrate and the suspension wire could occur. This allows the conclusion that the measurements were not limited by the setup below $100 \mathrm{~K}$. Nevertheless, resonance effects between 30 and $40 \mathrm{~K}$ were noticed. These are supposed to be relaxation processes in the test mass material. Further investigations are ongoing.

By using calcium fluoride and the results of this paper it would be possible to reduce the thermal noise of a GEO600-like mirror especially at low temperatures. Thus, calcium fluoride is a candidate for cryogenic optical components for gravitational wave detectors. A larger beam diameter or non-Gaussian laser beam would be needed to use the full low-noise potential of calcium fluoride due to its high thermoelastic noise contribution. Silicon which also has high $Q$-factors (up to several $10^{8}$ ) at low temperatures [40] and a lower thermoelastic noise contribution is opaque at the current wavelength of $1064 \mathrm{~nm}$. Optical components made of silicon would either demand a change of the laser to longer wavelengths or a change of the beamsplitter towards all reflective optics [41]. Especially the high optical transmittivity of calcium fluoride between 0.2 and $10 \mu \mathrm{m}$ makes it interesting for the use as a classical beamsplitter at low temperatures. Furthermore, the temperatures needed for cryogenic operation can easily be obtained from single-stage pulse-tube refrigerators [42] which provide cooling powers of several 10 Watts in the temperature region between $30 \mathrm{~K}$ and $100 \mathrm{~K}$.

This work was supported by the DFG (Deutsche Forschungsgemeinschaft) under contract SFB Transregio 7. The authors would like to thank Chr. Schwarz and P. Petruck for their support.

\section{References}

1. S. Hild et al., Classical Quant. Grav. 23, S643 (2006)

2. S.J. Waldman, Classical Quant. Grav. 23, S653 (2006)

3. F. Acernese et al., Classical Quant. Grav. 23, S635 (2006)

4. M. Ando et al., Classical Quant. Grav. 19, S1409 (2002)

5. V.B. Braginsky, V.P. Mitrofanov, V.I. Panov, Systems with Small Dissipation (The University of Chicago Press, Chicago, 1985)

6. S. Rowan, S. Reid for GEO600/Stranford Groups, (2006) LIGO-G050054-00-Z

7. S. Rowan, G. Cagnoli, P. Sneddon, J. Hough, R. Route, E.K. Gustafson, M.M. Fejer, V. Mitrofanov, Phys. Lett. A 265, 5 (2005)

8. K. Kuroda et al., Classical Quant. Grav. 20, S871 (2003)

9. P. Amico, L. Bosi, L. Carbone, L. Gammaitoni, F. Marchesoni, M. Punturo, F. Travasso, H. Vocca, Rev. Sci. Instrum. 73, 179 (2002)

10. Calcium fluoride product description, Korth $\mathrm{GmbH}$, www.korth.de

11. The results of the Perugia group are shown in the first year report of STREGA, available at: www.ego-gw.it/ILIASGW/documents/STREGAreport2005/ under task M1

12. K.A. Pandelisev, 2nd International Symposium on $157 \mathrm{~nm}$ Lithography, Dana Point, CA, USA, May 14-17, 2001

13. B.C. Stuart, M.D. Feit, A.M. Rubenchik, B.W. Shore, M.D. Perry, Phys. Rev. Lett. 74, 2248 (1995)

14. P.R. Saulson, Fundamentals of Interferometric Gravitational Wave Detectors (World Scientific, Singapore, 1994)

15. H.B. Callen, Th.A. Weldon, Phys. Rev. 83, 34 (1951)

16. T. Uchiyama, T. Tomaru, M.E. Tobar, D. Tatsumi, S. Miyoki, M. Ohashi, K. Kuroda, T. Suzuki, N. Sato, T. Haruyama, A. Yamamoto, T. Shintomi, Phys. Lett. A 261, 5 (1999)

17. S. Nietzsche, R. Nawrodt, A. Zimmer, M. Thürk, W. Vodel, P. Seidel, J. Phys. Conference Series 32, 445 (2006)

18. Z. Yan, L. Ju, S. Gras, C. Zhao, D.G. Blair, Eur. Phys. J. Appl. Phys. 30, 189 (2005)

19. K. Yamamoto, S. Miyoki, T. Uchiyama, H. Ishitsuka, M. Ohashi, K. Kuroda, T. Tomaru, N. Sato, T. Suzuki, T. Haruyama, A. Yamamoto, T. Shintomi, K. Numata, K. Waseda, K. Ito, K. Watanabe, Classical Quant. Grav. 21, S1075 (2004)

20. R. Nawrodt, A. Zimmer, S. Nietzsche, M. Thürk, W. Vodel, P. Seidel, Cryogenics 46, 718 (2006)

21. The tungsten wire was purchased at www.advent-rm.com

22. www.struers.com, DP-Suspension

23. www.lakeshore.com

24. S. Nietzsche, R. Nawrodt, A. Zimmer, R. Schnabel, W. Vodel, P. Seidel, Supercond. Sci. Technol. 19, S293 (2006)

25. www.sios.de, see device SP-S laserinterferometric vibrometer

26. Schott Lithotec AG, www.schott.com/lithotec, datasheet calcium fluoride

27. www.hellmaoptik.com

28. D.R. Huffman, M.H. Norwood, Phys. Rev. 117, 709 (1960)

29. D.N. Batchelder, R.O. Simmons, J. Chem. Phys. 41, 2324 (1964)

30. G.A. Slack, Phys. Rev. 122, 1451 (1961)

31. S.S. Todd, J. Am. Chem. Soc. 71, 4115 (1949)

32. www.ansys.com 
33. A. Zimmer, R. Nawrodt, R. Neubert, S. Nietzsche, M. Thürk, W. Vodel, P. Seidel, submitted to Phys. Rev. B

34. Yu. Levin, Phys. Rev. D 57, 659 (1998)

35. S.D. Penn, A. Ageev, D. Busby, G.M. Harry, A.M. Greatarsson, K. Numata, P. Willems, Phys. Lett. A 352, 3 (2006)

36. J.R. Smith, G. Cagnoli, D.R.M. Crooks, M.M. Fejer, S. Goßler, H. Lück, S. Rowan, J. Hough, K. Danzmann, Classical Quant. Grav. 21, S1091 (2004)

37. V.B. Braginski, M.L. Gorodetsky, S.P. Vyatchanin, Phys. Lett. A 271, 303 (2000)

38. V.B. Braginski, M.L. Gorodetsky, S.P. Vyatchanin, Phys. Lett. A 264, 1 (1999)
39. Y.T. Liu, K.S. Thorne, Phys. Rev. D 62, 122002-1 (2000)

40. D.F. McGuigan, C.C. Lam, R.Q. Gram, A.W. Hoffman, D.H. Douglass, H.W. Gutche, J. Low Temp. Phys. 30, 621 (1978)

41. A. Bunkowski, O. Burmeister, P. Beyersdorf, K. Danzmann, R. Schnabel, T. Clausnitzer, E.-B. Kley, A. Tünnermann, Opt. Lett. 29, 2342 (2004)

42. T. Koettig, R. Nawrodt, S. Moldenhauer, M. Thürk, P. Seidel, in Advances in Cryogenic Engineering, Vol. 51A, AIP Conference Proceedings, 2006, pp. 35-40 